\title{
A new method for solving the ground-state problem in arbitrary quantum wells: Application to electron-hole quasi-bound levels in quantum wells under high electric field
}

\author{
Jasprit Singh \\ Department of Electrical Engineering and Computer Science, The University of Michigan, Ann Arbor, \\ Michigan 48109
}

(Received 14 October 1985; accepted for publication 10 December 1985)

\begin{abstract}
A method based on the Monte Carlo technique and variational principle is developed to study the ground-state problem in arbitrary quantum wells. A technique is described to use this method to study quasi-bound states in systems. The method is applied to AlGaAs/GaAs quantum wells subjected to high electric fields. Advantages of this approach over the conventional variational approach are identified.
\end{abstract}

Novel crystal growth techniques have allowed fabrication of quantum wells and superlattices and have thus allowed tailoring of optical and electronic response of the structures. ${ }^{1-6}$ In order to understand the physical properties of the heterostructure device, one has to solve the eigenfunction problem of the charged particle in the quantum well. It is well known that exact analytic solutions to such problems are only available for simple potential structures such as square well, parabolic well, etc. and even in these structures, in general, in the presence of perturbations such as electric field, magnetic field, etc. the problem cannot be solved exactly. The variational approach has been widely used to solve the problem in general, but it suffers from the following serious drawback. The energy level and wave function depend upon the choice of the starting wave function (exponential, trigonometric, etc.). For simple quantum wells, such a choice is quite obvious, but for more complicated quantum wells (e.g., graded quantum wells, asymmetric quantum wells, wells in presence of high electric fields, etc.) such a choice often becomes very difficult to make and thus the results cannot be relied upon.

In this letter we describe a method based on the Monte Carlo method which is capable of solving the variational problem in an arbitrary quantum well under arbitrary perturbation. We will also describe a technique to calculate the quasi-bound states of quantum well and present results of these approaches for electron and hole ground-state levels under high electric field. This above problem is of great interest due to its application in light modulation. ${ }^{7-9}$ A number of experiments have been reported on this concept, but the quantitative understanding is not clear primarily because of the difficulty of solving the Schrödinger equation.

The variational approach to the ground-state problem is the following:

The Schrödinger equation to be solved is

$$
H \psi=\left(\frac{-\hbar^{2}}{2 m} \nabla^{2}+V(r)\right) \psi(r)=E \psi(r) \text {. }
$$

The ground-state energy $E_{0}$ is determined from the fact that

$$
E_{0}=\min \int \psi^{*} H \psi d r
$$

where the range of $\psi$ includes all arbitrary functions subject only to the normalization conditions

$$
\int|\psi|^{2} d r=1 \text {. }
$$

The variational principle has been used extensively in solving a number of problems in the area of quantum wells. ${ }^{10-13}$ But, as mentioned earlier, it is difficult to rely on the quantitative accuracy of a variational calculation, and in the particular case of electric field dependence of quasibound levels in a quantum well, the agreement with experiments is rather poor. ${ }^{13}$ Recently Miller et al. ${ }^{14}$ have used an approach based on numerical integration of Schrödinger equation and the agreement between our results based on Monte Carlo methods and theirs is very good.

In this letter we describe a method based on the Monte Carlo technique which finds a solution to the set of equations (1) - (3), but is not limited to any special choice of the wave function.

The Monte Carlo method. To solve the set of eqs. (1)(3) an arbitrary normalized wave function is chosen inside a fixed volume (see discussion on quasi-bound states), and randomly altered to minimize the energy associated with it.

This process is repeated (for $\sim 10^{4}$ iterations) until convergence is obtained. ${ }^{15}$ It is important to realize that the final solution is independent of the starting wave function although a judicious starting wave function can lower the number of iterations required to reach a stable solution.

Quasi-bound states. The practical computer aspects of going through steps (i) $-(v)$ above require that the physical space over which the wave function extends is finite. A square quantum well may be defined by

$$
V(x)=\left\{\begin{array}{l}
0 \text { if } \quad x_{0} \leqslant x \leqslant x_{0}+W \\
V_{0} \text { if } \quad x<x_{0} \text { or } x>x_{0}+W .
\end{array}\right.
$$

The space over which the bound state extends is typically $\sim W$ so that in the calculations one keeps information of distances a few times $W$. In case an electric field $E$ is applied to the quantum well, one has an additional potential given by

$$
\bar{V}(x)=e E x .
$$

In this case, the subband levels in the quantum well given by Eq. (4) can only be quasi-bound since for large enough $x$ away from the well the free-electron (hole) states are lower (higher) in energy than the subband levels. Since the Monte Carlo method described earlier will always find the wave function with the lowest energy, it is clear that the wave 
function will depend on the size of the system considered. To find the true quasi-bound state we exploit the fact that a quasi-bound state is confined near the well with very small probability away from the well. The following technique is used. An arbitrary potential is superimposed on the potentials defined by Eqs. (4) and (5). This arbitrary potential is defined by

$$
V_{A}= \begin{cases}V_{1} & x \leqslant x_{0}-L \text { or } x \geqslant x_{0}+W+L \\ 0 & \text { otherwise }\end{cases}
$$

where we choose $V_{1}>V_{0}$. The distance $L$ is gradually increased from 0 to higher values and the Monte Carlo approach described earlier is utilized to solve for the lowest energy wave function. As the distance $L$ is increased one expects that the ground-state wave function will show one of the two schematic behaviors discussed below.

In case of a true quasi-bound state, the energy associated with the wave function (as well as other properties such as shape, etc.) stabilizes at a value of $L=L c_{1}$ and remains essentially invariant till $L=L c_{2}$ after which the energy rapidly changes. In case there is no quasi-bound state, the energy (and other properties) associated with the lowest energy wave function changes uniformly as $L$ is changed. The physical argument behind this criterion is straightforward. ${ }^{15}$ If the ground state is a true quasi-bound state, it will be confined primarily near the quantum well region. $L c_{1}$ represents the distance up to which the wave function extends (of course, an extremely small fraction extends further into the barrier). Thus increase in $L$ does not significantly change the solution. However, beyond $L c_{2}$, the lowest energy states are the free band states. On the other hand, if the electric field is too high, one cannot talk about quasi-bound states [e.g., see Eq. (14) of Ref. 13]. This criterion is found to work extremely well for calculating the quasi-bound states as will be clear from the results presented below.

We have applied the above techniques to calculate the electron and hole ground-state quasi-subband levels and the associated wave functions for square wells formed from $\mathrm{Al}_{0.30} \mathrm{Ga}_{0.70} \mathrm{As} / \mathrm{GaAs}$. We assume band-gap discontinuities of $60 \%$ and $40 \%$ for the AlGaAs-GaAs interface conduction and valence bands, respectively. We assume electron and hole masses of 0.067 and 0.45 , respectively.

In Fig. 1 we show the electron and hole wave functions in a $100 \AA$ well when electric fields of (a) 0 and (b) 80 $\mathrm{kV} \mathrm{cm} \mathrm{cm}^{-1}$ are applied to the quantum well. Both cases in Fig. 1 satisfy the conditions required for a quasi-bound state [case (a) is a bound state]. In Fig. 2 we show the case when the applied electric field is $160 \mathrm{kV} \mathrm{cm}^{-1}$. In this case we show the wave functions calculated with $V_{1}$ in Eq. (6) chosen to be $1000 \mathrm{meV}$ for electrons and for holes (the results are independent of the choice of $V_{1}$ as long as $\left.V_{1}>V_{0}\right) ; V_{0}=216 \mathrm{meV}$ for electrons and $144 \mathrm{meV}$ for holes. The $L$ defined by Eq. (6) changes from 60 to $120 \AA$ in steps of $20 \AA$ for the four cases shown. It is clear that for this electric field, the hole wave function is not a quasi-bound state, but an unbound state. This is consistent with the following physical observation. The hole wave function penetrates $\sim 50 \AA$ beyond the barrier at $x=x_{0}+W_{0}$. At $E=160 \mathrm{kV} \mathrm{cm}^{-1}$ the shift in the band position over $50 \AA$ is $80 \mathrm{meV}$. Since the difference between the hole subband and
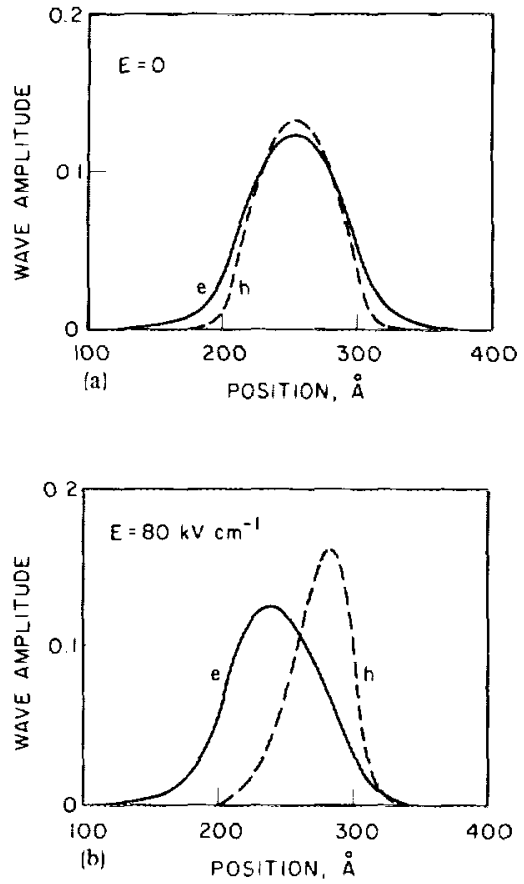

FIG. 1. Electron and hole wave functions in a $100 \AA$ quantum well in presence of (a) electric field $E=0$; (b) $E=80 \mathrm{kV} \mathrm{cm}-1$.

the barrier at $x=w_{0}$ is also $\sim 80 \mathrm{meV}$, at such high fields, the wave function becomes unbound.

Finally in Fig. 3 we show the variation of the subband energy (difference between electron and hole energies) for a 100 and a $30 \AA$ quantum well as a function of the applied electric field. Detailed results on the tunneling of electrons and holes using the above techniques as well as its effect on photoluminescence quenching will be published elsewhere ${ }^{15}$ Here we would like to point out some of the highlights of our results: (a) at high fields the results are quite different from those calculated using the variational approach. ${ }^{13}$ In particular the electric field at which the levels become unbound is found to be much lower. For example, if
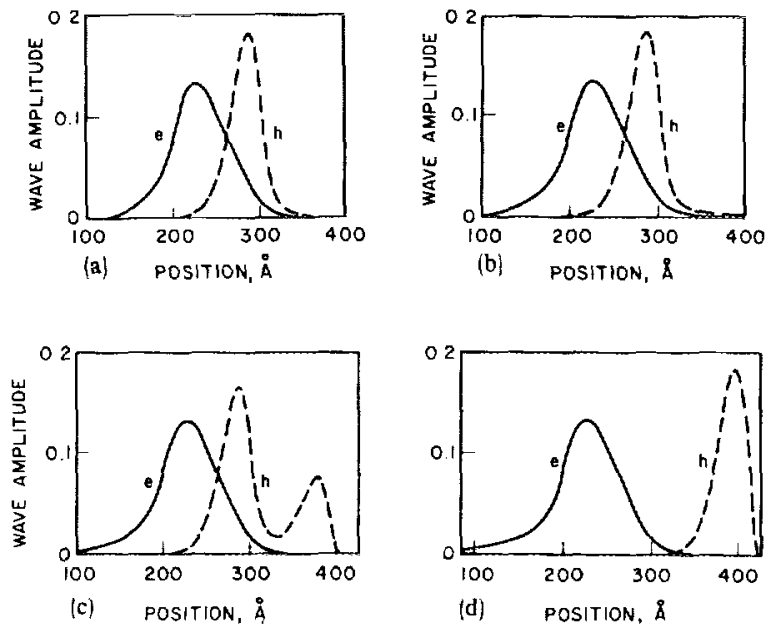

FIG. 2. Monte Carlo results for the electron and hole wave functions in presence of an electric field of $160 \mathrm{kV} \mathrm{cm}^{-1}$. At this high field, the hole state is not a quasi-bound state, since the hole wave function does not reach a stable state as the artificial barrier described in the text is removed from 60 to $120 \AA$ in steps of $20 \AA$. 


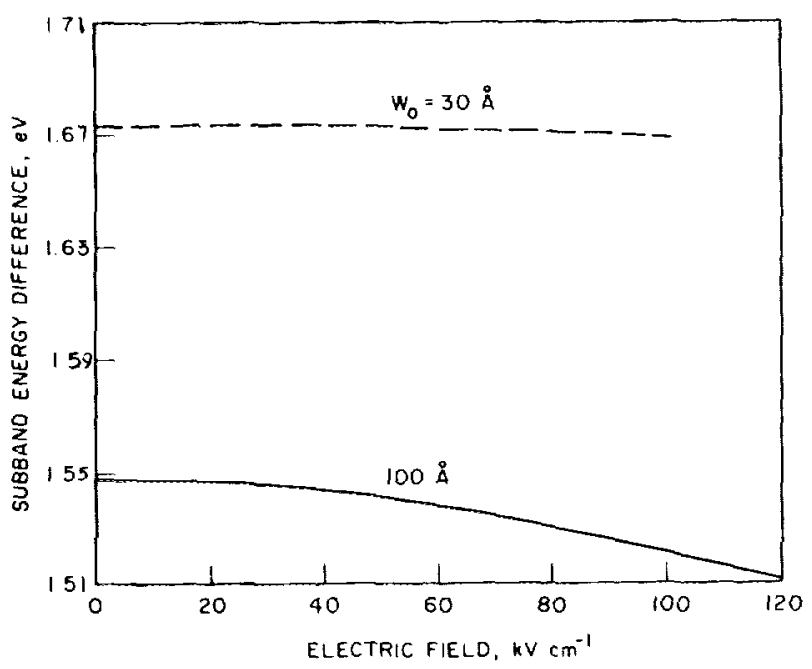

FIG. 3. Variation of the subband energy difference as a function of electric field for 100 and $30 \AA$ quantum wells.

a $85: 15$ discontinuity were assumed for the $100 \AA$ quantum well, the hole levels become unbound at $45 \mathrm{kV} / \mathrm{cm}$ according to the Monte Carlo calculations, while the conventional variational approach suggests that quasi-bound states exist up to $100 \mathrm{kV} / \mathrm{cm} .{ }^{13}$ We believe that this difference arises because in the conventional variational approach an exponentially decaying wave function is chosen, even though the field is so high that it is meaningless to talk of quasi-bound states. The implication of these results for light modulation and PL studies will be discussed elsewhere. ${ }^{15}$ (b) We also point out that this method can be applied to arbitrary shaped quantum wells which can provide valuable insight into de- signing structures with tailored optical response in presence of electric field.

In conclusion, we have presented a versatile Monte Carlo method to calculate bound and quasi-bound levels in arbitrary potentials and applied it to the problem of electron hole levels in quantum wells under high electric field.

The author is grateful for valuable discussions with Dr. K. K. Bajaj and Dr. P. Yu.

'C. E. C. Wood, in Physics of Thin Films, edited by George Haas and M. H. Francombe (Academic, New York, 1980), Vol. 11, p. 35.

${ }^{2}$ A. Y. Cho, Thin Solid Films 100, 291 (1983).

${ }^{3}$ J. R. Arthur, Surf, Sci. 43, 449 (1974).

${ }^{4}$ See for example, articles published in Proceedings of the International Conference on Metalorganic Vapor Phase Epitaxy, edited by J. F. Bonfils, S. J. Irvine, and J. B. Mullin (North-Holland, Amsterdam, 1981).

${ }^{5}$ C. Weisbuch, R. Dingle, P. M. Pettroff, A. C. Gossard, and W. Wiegmann, Appl. Phys. Lett. 38, 840 (1981).

${ }^{6}$ J. N. Schulman and T. C. McGill, Phys. Rev. Lett. 39, 1680 (1977).

'E. E. Mendez, G. Bastard, L. L. Chang, L. Esaki, H. Morkoç, and R. Fischer, Phys. Rey. B 26, 7101 (1982).

${ }^{8}$ T. H. Wood, C. A. Burrus, D. A. B. Miller, D. S. Chemla, T. C. Dammen, A. C. Gossard, and W. Wiegman, Appl. Phys. Lett. 44, 16 (1984).

${ }^{9}$ W. D. Goodhue, B. E. Burke, K. B. Nichols, G. M. Metze, and G. D. Johnson, presented at 6th MBE Workshop Aug. 14-16, 1985, Minneapolis, $\mathrm{MN}$.

${ }^{10}$ G. Bastard, Phys. Rev. B 24, 4714 (1981).

"C. Mailhiot, Y-C. Chang, and T. C. McGill, Phys. Rev. B 26, 4449 (1982).

${ }^{12}$ R. Green and K. K. Bajaj, Solid State Commun. 45, 025 (1983).

${ }^{13}$ G. Bastard, E. E. Mendez, L. L. Chang, and L. Esaki, Phys. Rev. B 28, 3241 (1983).

${ }^{14}$ D. A. B. Miller, D. S. Miller, D. S. Chemla, T. C. Damen, A. C. Gossard, W. Wiegmann, T. H. Wood, and C. A. B. Works, Phys. Rev. B 32, 1043 (1985)

15J. Singh (unpublished). 